\title{
Prognostic Nutritional Index Predicts Life Expectancy of Patients with End-Stage Oral Cancer: A Retrospective Study
}

\author{
Atsushi Abe ${ }^{1 *}$, Kenichi Kurita², Hiroki Hayashi', Masashi Minagawa1 \\ ${ }^{1}$ Department of Oral and Maxillofacial Surgery, Nagoya Ekisaikai Hospital, Nagoya, Japan \\ ${ }^{2}$ Department of Oral and Maxillofacial Surgery, School of Dentistry, Aichi-gakuin University, Nagoya, Japan \\ Email: *atsushi.a@ekisai.or.jp
}

How to cite this paper: Abe, A., Kurita, K., Hayashi, H. and Minagawa, M. (2018) Prognostic Nutritional Index Predicts Life Expectancy of Patients with End-Stage Oral Cancer: A Retrospective Study. Surgical Science, 9, 487-495.

https://doi.org/10.4236/ss.2018.912056

Received: November 12, 2018

Accepted: December 18, 2018

Published: December 21, 2018

Copyright $\odot 2018$ by authors and Scientific Research Publishing Inc. This work is licensed under the Creative Commons Attribution International License (CC BY 4.0).

http://creativecommons.org/licenses/by/4.0/

\begin{abstract}
Background: Generally, clinicians do not accurately estimate life expectancy in terminally ill patients with cancer. Aim: To evaluate the value of the Prognostic Nutritional Index (PNI) for accurately estimating the life expectancy of patients with end-stage oral cancer. Design: A longitudinal section study. Setting/participants: Fifteen patients (12 men; mean age: 71.7 years) who died of oral cancer between 2005 and 2014 (the terminal group) were included. The mean PNI values at the initial visit and at 3,2, and 1 months before the deaths were comparatively analyzed. Results: The mean follow-up period was 133 days. At the initial examination, the PNI values were $49.1 \pm 4.5$ ( $\mathrm{p}=0.6723$ ). The PNI value of the terminal group was $35.6 \pm 5.1$ at 2 months before death and $28.6 \pm 3.0$ at 1 month before death. The PNI values at 3,2, and 1 months before death in the terminal group significantly differed from each other and from that at the initial visit and steadily decreased until death. Conclusions: Our findings suggest the utility of PNI as a prognostic index in patients with end-stage oral cancer patients. Furthermore, the PNI should be routinely considered in the nutritional management of patients with oral cancer nearing death.
\end{abstract}

\section{Keywords}

Life Expectancy, Nutrition Assessment, Oral Cancer, Prognosis, Retrospective Studies

\section{Introduction}

Accurate estimation of life expectancy in terminally ill patients with cancer is important from both the clinician's and the patient's perspectives. However, 
studies have revealed that clinicians do not accurately estimate the time to death [1] [2] [3] [4] [5], making it difficult for the patients and their families to prepare themselves. Although several indices and scores exist for evaluating the life expectancy [6] [7], most of these include subjective components in the calculation, such as the presence of edema, anorexia, or delirium, which makes the objective assessment prone to inaccuracy. An objective scoring method to accurately predict the patient survival has therefore been sought. For this purpose, the Prognostic Nutritional Index (PNI) established by Onodera and Buzby [8] [9] can be used to objectively evaluate the prognosis in a range of patients with cancer. The PNI is calculated using a simple formula that is based on 2 routine serum measurements. Initially, the PNI was used to quantitatively estimate the postoperative complications in general surgical patients, with a PNI $>45$ indicating cancer surgery; 45 - 40 suggesting the need for caution during surgery; and $<40$ contraindicating surgery [8]. Regarding dental surgery, a few reports have been considered to enable prognostic prediction in the nutritional field [10] [11]. Recently, the practicality of PNI has been confirmed in an array of terminally ill patients [12] with cancer of the cervix [13], esophagus [14], stomach [15] [16], colorectum [17], and pleura [18]. Nevertheless, it remains unknown whether the index can serve as a predicative tool in patients with end-stage oral cancer, as mastication and deglutition functions are lost and the dietary intake is decreased. These changes negatively affect the nutritional and immunological status, thereby rapidly worsening the overall health and eventually leading to the patient's death. Subjective global assessment (SGA) for the nutritional screening of patients with cancer is available [19] [20]. However, this index is regarded as a qualitative indicator because of the subjective judgment involved with it. Because a patient's life expectancy and the quality of life can be improved if a health professional performs proper nutritional management, it is necessary to optimize the nutritional assessment and the prediction of survival time. The present study explores the use of the PNI in predicting the life expectancy of patients with end-stage oral cancer.

\section{Methods}

\subsection{Study Design}

This longitudinal section study was performed after obtaining ethical approval and in-formed consent. The study was approved by the Ethics Committee of the Nagoya Ekisai Hospital (Approval No. 2017-011). The present study was conducted in accordance with The Code of Ethics of the World Medical Association (Declaration of Helsinki) for experiments involving human subjects. Verbal informed consent was obtained from all participants and/or their families. The cases were retrospectively analyzed based on the available medical records. This study was conducted on 148 oral cancer patients who were provided radical treatment in the Department of Oral and Maxillofacial Surgery, Nagoya Ekisaikai Hospital between January 2005 and April 2014. Among the patients who re- 
ceived radical treatment, we excluded the following: 1) 98 patients without the recurrence metastatic after radical treatment. 2) 35 patients who remitted after performing salvage operation or CRT. 15 patients with oral cancer who eventually died at the Nagoya Ekisaikai Hospital between April 2005 and March 2014 after receiving resection and reconstruction surgery were selected. A patient diagnosed as having oral squamous cell carcinoma and receiving radiotherapy and chemotherapy before the surgery was excluded from group.

\subsection{Data Collection}

The following data were collected from the patient medical records: sex, age, site of primary tumor, C-reactive protein (CRP) levels, serum albumin levels, and peripheral lymphocyte count measured at the initial visit and at various time points during the follow-up period.

\subsection{Tumor Staging}

Tumor staging was performed according to the TNM classification method of the International Union against Cancer [21] during the initial visit.

Serum parameter measurement and data analysis.

The serum levels of CRP and albumin and peripheral lymphocyte count were estimated using the standard laboratory procedures. In terminally ill patients, the measurements were performed at the initial visit and routinely thereafter at every week, including at approximately 3,2, and 1 months before their deaths. PNI value was calculated using the following formula:

$$
\begin{aligned}
P N I= & {[10 \times \text { serum albumin level }(\mathrm{g} / \mathrm{dL})] } \\
& +\left[0.005 \times \text { total peripheral lymphocyte } \operatorname{count}\left(\operatorname{per} \cdot \mathrm{mm}^{3}\right)\right]
\end{aligned}
$$

\subsection{Statistical Analysis}

The weekly PNI data were analyzed using Shapiro-Wilk test to ascertain whether they were normal distribution we performed iteration measurement with 1-way layout analysis of variance and examined the factor effect based on the week number. After proving the significant factor effect by the number in a week, we compared the effect between the standards by multiple comparisons using the Bonferroni method. The statistical significance standard was set at $5 \%$.

\section{Results}

The participants comprised 12 men and 3 women aged 55 - 89 years (mean age: $71.7 \pm 8.5$ years). The patients presented with a primary tumor on the tongue ( $\mathrm{n}$ $=8)$, lower jaw $(\mathrm{n}=4)$, upper jaw $(\mathrm{n}=1)$, oral floor $(\mathrm{n}=1)$, or buccal mucosa ( $\mathrm{n}$ $=1)$, and their disease was diagnosed at stage I $(n=2)$, stage II $(n=4)$, stage III $(\mathrm{n}=6)$, or stage IV $(\mathrm{n}=3)$.

The detailed characteristics of the terminal participants are provided in $\mathrm{Ta}$ ble 1 . 
Table 1. Patient characteristics.

\begin{tabular}{|c|c|c|}
\hline Factor & Group & Overall \\
\hline $\mathrm{n}$ & & 15 \\
\hline Age (years) & & $71.7 \pm 8.5$ \\
\hline \multirow[t]{2}{*}{$\operatorname{Sex}(n)$} & Male & 12 \\
\hline & Female & 3 \\
\hline \multirow[t]{4}{*}{ site of primary } & Tongue & 9 \\
\hline & Upper iaw & 1 \\
\hline & Lower jaw & 4 \\
\hline & Buccal mucosa & 1 \\
\hline \multirow[t]{4}{*}{$\mathrm{T}$ classification } & $\mathrm{T} 1$ & 3 \\
\hline & $\mathrm{T} 2$ & 7 \\
\hline & $\mathrm{T} 3$ & 5 \\
\hline & $\mathrm{T} 4$ & 0 \\
\hline \multirow[t]{4}{*}{$\mathrm{N}$ classification } & No & 7 \\
\hline & N1 & 5 \\
\hline & $\mathrm{N} 2$ & 2 \\
\hline & N3 & 1 \\
\hline \multirow[t]{4}{*}{ Stage $(\mathrm{n})$} & I & 2 \\
\hline & II & 4 \\
\hline & III & 6 \\
\hline & IV & 3 \\
\hline Observation period & & $583.5 \pm 336.1$ \\
\hline
\end{tabular}

In the Shapiro-Wilk test, the PNI data of each month was regularly distributed.

The mean PNI value at the initial visit was $50.1 \pm 7.9$. However, the values gradually decreased over a period of time, with the mean values at 3,2 , and 1 months before the death being $41.4 \pm 6.2,35.6 \pm 5.1$, and $28.5 \pm 3.0$ (Figure 1). Furthermore, in the terminal group, the mean PNI values at 3 versus 2 months $(\mathrm{p}=0.000021)$ and 2 versus 1 month $(\mathrm{p}=0.000028)$ before death were significantly different from each other. The mean value at 3 months before death was significantly lower than that obtained during the initial visit $(p=0.001173)$ (Figure 1). PNI value at decreased before 1 month $(\mathrm{p}<0.05), 2$ months as compared with the initial diagnosis in 3 months of death ( $p<0.01)$. Using iteration measurement with 1-way layout analysis of variance, we showed the significant factor effect by the number in month passed $(\mathrm{p}<0.05)$. Multiple comparisons by the Bonferroni method demonstrated significant differences in all combinations between the initial diagnosis and at a month before death.

\section{Discussion}

\subsection{Main Findings}

In this study, we established that the PNI is a suitable tool for accurately predicting the life expectancy of patients with end-stage oral cancer. The disease has 


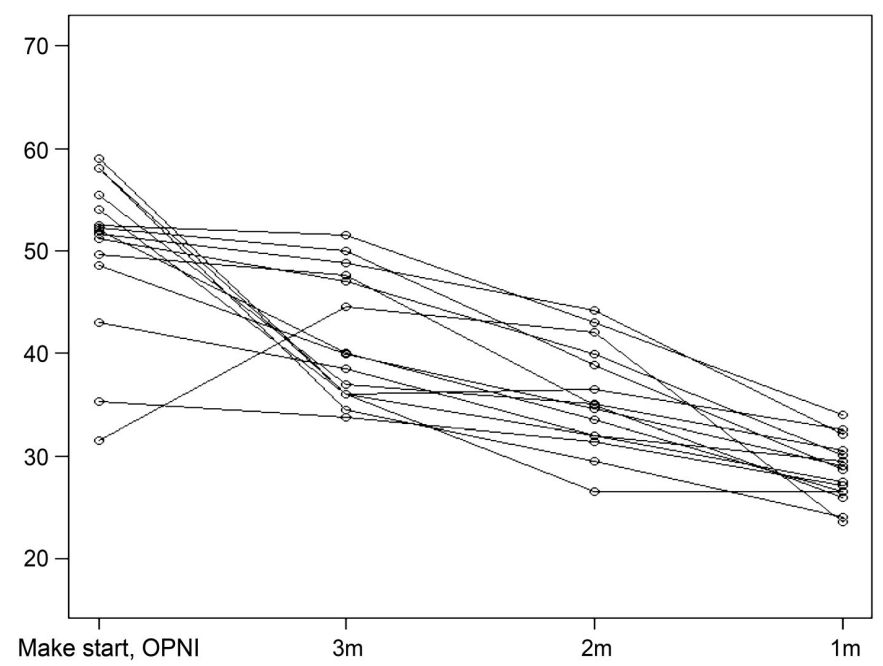

Figure 1. Prognostic Nutritional Index (PNI) values of oral cancer patients who eventually died from the disease. The PNI values were 50.1, $41.4,35.6$, and 28.5 at the time of the first examination and at 3 months, 2 months, 1 month, and 1 week before death, showing a gradual decrease over a period of time. In this study, the mean PNI values of the 15 study patients were 28.5 and 35.6 at 1 and 2 months before death, respectively.

a high mortality rate, with 5-year survival rate being approximately 50\% [22]. In light of this low survival rate, it is imperative to accurately predict the life expectancy of the patients. If an early prognosis can be established by a trained health professional, the patients can spend their remaining time with an appropriate focus and make timely preparations for their death together with their loved ones. To date, the nutritional and overall statuses of patients with head and neck cancer have been evaluated using anthropometric indices, laboratory parameters, dietary intake assessments, and clinical scores [23] [24]. Various prognostic tools such as the Karnofsky performance status, Eastern Cooperative Oncology Group performance status, Palliative Performance Scale, Palliative Prognostic Score, and Palliative Prognostic Index were used interchangeably [25]. However, all these tools included subjective components. The PNI [8] [9] originally developed for general surgical patients to predict postoperative complications has recently gained attention as a more objective measurement tool for the prognosis of a broad range of terminally ill patients with cancer [12]-[18]. The merit of PNI lies in the fact that it can be easily calculated from only 2 serum markers: serum albumin value and peripheral lymphocyte count. The index can therefore be computed as a part of the routine hematological investigation. Serum albumin is a protein with a half-life of 20 days, and it is compounded by the liver; hence, it reflects the long-term variations in the whole-body nutritional status. In healthy individuals, one-third to half of serum albumin exists in the intravascular compartment and the remaining is distributed in the musculature and viscus. General dyscrasia, dysnephria, liver dysfunction, and poor nutrition are 
some of the reasons for decrease in serum albumin levels in patients with oral cancer. Another factor is that the oral cavity is an important organ in nutrition, signifying that any abnormalities affecting the masticating and swallowing functions exert a measurable influence on the serum albumin value. Metabolic changes in patients with cancer also occur because of tumors producing aberrant amounts and profiles of cytokines. For instance, interleukin-6, interleukin-1, and tumor necrosis factor alpha levels are typically increased, thereby lowering the skeletal muscle and body fat, which in turn is accompanied by a decrease in immunocompetence [26] [27] [28].

\subsection{What This Study Adds}

We discerned that the PNI value significantly and steadily decreased at 3,2, and 1 months before death, thereby providing an accurate measure of a patient's survival time. It was previously documented that patients with gastrointestinal cancer present with a mean PNI of 40.4, 39.2, and 35.4 at 3, 2, and 1 months before death, respectively [12]. These values differ from the mean PNI values observed in our study: $41.4,35.6$, and 28.5 at 3, 2, and 1 months before death, respectively. This deviation indicates that the nutritional status of patients with oral cancer is somewhat more compromised at 2 and 1 months before death when compared with that of patients with gastrointestinal cancer. In fact, a PNI value of 28.5 at 1 month before death, as estimated in the present study, corresponds to a value of 29.8 at 3 days before death in patients with gastrointestinal cancer, as reported previously [12]. This comparison reveals that patients with oral cancer do survive, albeit under severe nutritional deprivation, for as long as patients with gastrointestinal cancer have a slightly higher PNI. To generate a more accurate estimate of patient survival time, the PNI values for patients with oral cancer nearing their death should be verified using a larger number of participants and more frequent measurements at time points closer to death. In addition, to enhance the remaining quality of life, future research should focus on deciphering how the PNI can be applied to improve the nutritional management in the final stages of the patient's survival.

\subsection{Study Limitations}

Although participants were carefully selected to avoid bias, the study had a small sample size, thereby limiting its generalizability.

\section{Conclusion and Limitations}

Clinicians should consider using the PNI value when estimating life expectancy in patients with end-stage oral cancer. Considering the particular importance of appropriate nutritional management in these patients at the end stage of the disease, more research is necessary to investigate how the PNI value can be used in these patients to improve the quality of life near death. 


\section{Acknowledgements}

The present research did not receive any specific grant from funding agencies in the public, commercial, or not-for-profit sectors.

\section{Conflicts of Interest}

The authors declare that there is no conflict of interest.

\section{References}

[1] Christakis, N.A. and Lamont, E.B. (2000) Extent and Determinants of Error in Doctors' Prognoses in Terminally Ill Patients: Prospective Cohort Study. BMJ, 320, 469-472. https://doi.org/10.1136/bmj.320.7233.469

[2] Glare, P., Virik, K., Jones, M., Hudson, M., Eychmuller, S., Simes, J., et al. (2003) A Systematic Review of Physicians' Survival Predictions in Terminally Ill Cancer Patients. BMJ, 327, 195-198. https://doi.org/10.1136/bmj.327.7408.195

[3] Leung, K.M., Hopman, W.M. and Kawakami, J. (2012) Challenging the 10-Year Rule: The Accuracy of Patient Life Expectancy Predictions by Physicians in Relation to Prostate Cancer Management. Canadian Urological Association Journal = Jour nal de I Association des Urologues du Canada, 6, 367-373.

[4] Trevino, K.M., Zhang, B., Shen, M.J. and Prigerson, H.G. (2016) Accuracy of Advanced Cancer Patients' Life Expectancy Estimates: The Role of Race and Source of Life Expectancy Information. Cancer, 122, 1905-1912.

https://doi.org/10.1002/cncr.30001

[5] Viganò, A., Dorgan, M., Bruera, E. and Suarez-Almazor, M.E. (1999) The Relative Accuracy of the Clinical Estimation of the Duration of Life for Patients with End of Life Cancer. Cancer, 86, 170-176. https://doi.org/10.1002/(SICI)1097-0142(19990701)86:1<170::AID-CNCR23>3.0.C $\mathrm{O} ; 2-\mathrm{S}$

[6] Morita, T., Tsunoda, J., Inoue, S. and Chihara, S. (1999) The Palliative Prognostic Index: a Scoring System for Survival Prediction of Terminally Ill Cancer Patients. Supportive Care in Cancer, 7, 128-133. https://doi.org/10.1007/s005200050242

[7] Pirovano, M., Maltoni, M., Nanni, O., Marinari, M., Indelli, M., Zaninetta, G., et al. (1999) A New Palliative Prognostic Score: A First Step for the Staging of Terminally Ill Cancer Patients. Italian Multicenter and Study Group on Palliative Care. Journal of Pain \& Symptom Management, 17, 231-239. https://doi.org/10.1016/S0885-3924(98)00145-6

[8] Buzby, G.P., Mullen, J.L., Matthews, D.C., Hobbs, C.L. and Rosato, E.F. (1980) Prognostic Nutritional Index in Gastrointestinal Surgery. American Journal of Surgery, 139, 160-167. https://doi.org/10.1016/0002-9610(80)90246-9

[9] Onodera, T., Goseki, N. and Kosaki, G. (1984) [Prognostic Nutritional Index in Gastrointestinal Surgery of Malnourished Cancer Patients]. Nihon Geka Gakkai Zasshi, 85, 1001-1005. (In Japanese).

[10] Guo, C.B., Ma, D.Q. and Zhang, K.H. (1994) Applicability of the General Nutritional Status Score to Patients with Oral and Maxillofacial Malignancies. International Journal of Oral \& Maxillofacial Surgery, 23, 167-169. https://doi.org/10.1016/S0901-5027(05)80294-2

[11] Guo, C.B., Zhang, W., Ma, D.Q., Zhang, K.H. and Huang, J.Q. (1996) Hand Grip Strength: An Indicator of Nutritional State and the Mix of Postoperative Complica- 
tions in Patients with Oral and Maxillofacial Cancers. British Journal of Oral \& Maxillofacial Surgery, 34, 325-327. https://doi.org/10.1016/S0266-4356(96)90012-1

[12] Nakamura, Y., Nagao, J., Saida, Y., Watanabe, M., Okamoto, Y., Asai, K., et al. (2013) Use of the Prognostic Nutritional Index to Predict Clinical Outcomes of Patients with Terminal Stage Cancer. Palliative Care Research, 8, 199-202.

[13] Haraga, J., Nakamura, K., Omichi, C., Nishida, T., Haruma, T., Kusumoto, T., et al. (2016) Pretreatment Prognostic Nutritional Index Is a Significant Predictor of Prognosis in Patients with Cervical Cancer Treated with Concurrent Chemoradiotherapy. Molecular \& Clinical Oncology, 5, 567-574. https://doi.org/10.3892/mco.2016.1028

[14] Feng, J.F. and Chen, Q.X. (2014) Significance of the Prognostic Nutritional Index in Patients with Esophageal Squamous Cell Carcinoma. Therapeutics \& Clinical Risk Management, 10, 1-7. https://doi.org/10.2147/TCRM.S56159

[15] Nozoe, T., Ninomiya, M., Maeda, T., Matsukuma, A., Nakashima, H. and Ezaki, T. (2010) Prognostic Nutritional Index: A Tool to Predict the Biological Aggressiveness of Gastric Carcinoma. Surgery Today, 40, 440-443. https://doi.org/10.1007/s00595-009-4065-y

[16] Migita, K., Takayama, T., Saeki, K., Matsumoto, S., Wakatsuki, K., Enomoto, K., et al. (2013) The Prognostic Nutritional Index Predicts Long-Term Outcomes of Gastric Cancer Patients Independent of Tumor Stage. Annals of Surgical Oncology, 20, 2647-2654. https://doi.org/10.1245/s10434-013-2926-5

[17] Nagata, T., Nakase, Y., Nakamura, K., Sougawa, A., Mochiduki, S., Kitai, S., et al. (2016) Prognostic Impact of a Nutritional Index Including Muscle Volume in Stage 4 Colorectal Cancer. In Vivo, 30, 885-891. https://doi.org/10.21873/invivo.11009

[18] Yao, Z.H., Tian, G.Y., Wan, Y.Y., Kang, Y.M., Guo, H.S., Liu, Q.H., et al. (2013) Prognostic Nutritional Index Predicts Outcomes of Malignant Pleural Mesothelioma. Journal of Cancer Research \& Clinical Oncology, 139, 2117-2123. https://doi.org/10.1007/s00432-013-1523-0

[19] Bauer, J., Capra, S. and Ferguson, M. (2002) Use of the Scored Patient-Generated Subjective Global Assessment (PG-SGA) as a Nutrition Assessment Tool in Patients with Cancer. European Journal of Clinical Nutrition, 56, 779-785. https://doi.org/10.1038/sj.ejcn.1601412

[20] Martin, L., Watanabe, S., Fainsinger, R., Lau, F., Ghosh, S., Quan, H., et al. (2010) Prognostic Factors in Patients with Advanced Cancer: Use of the Patient-Generated Subjective Global Assessment in Survival Prediction. Journal of Clinical Oncology: Official Journal of the American Society of Clinical Oncology, 28, 4376-4383. https://doi.org/10.1200/JCO.2009.27.1916

[21] International Union against Cancer (2010) TNM Classification of Malignant Tumours. 7th Edition, Wiley-Blackwell, Hoboken.

[22] Sciubba, J.J. (2009) End of Life Considerations in the Head and Neck Cancer Patient. Oral Oncology, 45, 431-434. https://doi.org/10.1016/j.oraloncology.2008.06.001

[23] Dechaphunkul, T., Martin, L., Alberda, C., Olson, K., Baracos, V. and Gramlich, L. (2013) Malnutrition Assessment in Patients with Cancers of the Head and Neck: A Call to Action and Consensus. Critical Reviews in Oncology/ Hematology, 88, 459-476. https://doi.org/10.1016/j.critrevonc.2013.06.003

[24] Prevost, V., Joubert, C., Heutte, N. and Babin, E. (2014) Assessment of Nutritional Status and Quality of Life in Patients Treated for Head and Neck Cancer. European 
Annals of Otorhinolaryngology, Head \& Neck Diseases, 131, 113-120. https://doi.org/10.1016/j.anorl.2013.06.007

[25] de Kock, I., Mirhosseini, M., Lau, F., Thai, V., Downing, M., Quan, H., et al. (2013) Conversion of Karnofsky Performance Status (KPS) and Eastern Cooperative Oncology Group Performance Status (ECOG) to Palliative Performance Scale (PPS), and the Interchangeability of PPS and KPS in Prognostic Tools. Journal of Palliative Care, 29, 163-169.

[26] Dülger, H., Alici, S., Sekeroğlu, M.R., Erkog, R., Ozbek, H., Noyan, T., et al. (2004) Serum Levels of Leptin and Proinflammatory Cytokines in Patients with Gastrointestinal Cancer. International Journal of Clinical Practice, 58, 545-549. https://doi.org/10.1111/j.1368-5031.2004.00149.x

[27] Krzystek-Korpacka, M., Matusiewicz, M., Diakowska, D., Grabowski, K., Blachut, K., Kustrzeba-Wojcicka, I., et al. (2007) Impact of Weight Loss on Circulating IL-1, IL-6, IL-8, TNF-alpha, VEGF-A, VEGF-C and Midkine in Gastroesophageal Cancer Patients. Clinical Biochemistry, 40, 1353-1360. https://doi.org/10.1016/j.clinbiochem.2007.07.013

[28] Richey, L.M., George, J.R., Couch, M.E., Kanapkey, B.K., Yin, X., Cannon, T., et al. (2007) Defining Cancer Cachexia in Head and Neck Squamous Cell Carcinoma. Clinical Cancer Research: An Official Journal of the American Association for Cancer Research, 13, 6561-6567. https://doi.org/10.1158/1078-0432.CCR-07-0116 